\title{
Preference of Prior for Two-Component Mixture of Lomax Distribution
}

\author{
Faryal Younis ${ }^{1}$, Muhammad Aslam ${ }^{2}$, M. Ishaq Bhatti ${ }^{3}$, , (1) \\ ${ }^{1}$ School of Applied Sciences and Humanities, National University of Technology, Islamabad, Pakistan \\ ${ }^{2}$ Department of Mathematics and Statistics, Riphah International University, Islamabad, Pakistan \\ ${ }^{3}$ La Trobe University, Melbourne, Australia
}

A R T I LE I N F
Article History
Received 26 Sep 2020
Accepted 16 Jun 2021
Keywords
Mixture of Lomax distribution
Censored sampling
Elicitation of hyperparameter
Bayes estimator
Posterior risk
Loss function

\begin{abstract}
Recently, El-Sherpieny et al., (2020), suggested Type-II hybrid censoring method for parametric estimation of Lomax distribution (LD) without due regard being given to the choice of priors and posterior risk associated with the model. This paper fills this gap and derived the new LD model with minimum posterior risk for the selection of priors. It derives a closed form expression for Bayes estimates and posterior risks using square error loss function (SELF), weighted loss function (WLF), quadratic loss function (QLF) and DeGroot loss function (DLF). Prior predictive approach is used to elicit the hyperparameters of mixture model. Analysis of Bayes estimates and posterior risks is presented in terms of sample size $(n)$, mixing proportion $(p)$ and censoring rate $\left(t_{0}\right)$, with the help of simulation study. Usefulness of the model is demonstrated on applying it to simulated and real-life data which show promising results in terms of better estimation and risk reduction.
\end{abstract}

(C) 2021 The Authors. Published by Atlantis Press B.V. This is an open access article distributed under the CC BY-NC 4.0 license (http://creativecommons.org/licenses/by-nc/4.0/).

\section{INTRODUCTION}

Finite mixture distributions have been extensively used in different fields of human endeavors to model diverse economic activities like in COVID-19 pandemics and global financial crises. Use of mixture distributions while dealing with lifetime distributions is recommended because such distributions always have several subpopulations. Mixture distributions are in extensive use for a wide variety of practical situations, e.g., in physical sciences, chemical, biological sciences, medicine, psychiatry, genetics, astronomy, marketing, economics, finance, insurance, engineering and other fields. Since there are more parameters in a mixture distribution than single-component distribution thus results produced by a mixture distribution will produce a better fit, and characterization of both variability and uncertainty.

The analysis of mixture models under Bayesian framework has developed a significant interest among statisticians. For example, Ali [1], Aslam [2], Corbelini et al. [3], Majeed and Aslam [4], Sultana et al. [5], ZeinEldin et al. [6], El-Sherpieny et al. [7] and Ibrahim and Yousof [8] are among others. Some of these authors have described the Bayesian analysis of 2-component mixture of inverted exponential distribution under quadratic loss function (QLF). For example, note that Majeed and Aslam [4] described the Bayesian analysis of 2-component mixture of inverted exponential distribution under QLF. Ali [1] described the 2-component mixture of the inverse Rayleigh distributions under Bayesian framework. Sultana et al. [5,9] presented statistical analysis of mixture of Gumbel Type II and mixture of inverted exponential distributions under Bayesian approach.

Originally, the Lomax distribution (LD) was introduced by Lomax [10] for analysis of business failure data. LD has been used in reliability modeling and life-testing studies by Hassan and Al-Ghamdi [11] and applied to income and wealth distribution by Harris [12] size of cities, to firm size by Corbelini et al. [3] to queuing problems and size of computer files by Holland et al. [13]. LD has been useful for modeling and analyzing the lifetime data in engineering, biological and medical sciences, etc. For more details on LD see [14-17]. Several types of data are encountered in everyday life, regarding simple data, grouped data, truncated data, censored data and progressively censored data. Censoring is a form of missing data and is inevitable attribute of lifetime applications as an experiment cannot continue for an infinite time due to cost and available time limitations. Different censoring schemes include left, right, interval, Type-I and Type-II censoring. A valuable account of censoring is given in by Kalbfleisch and Prentice [18]. Recent studies by El-Sherpieny et al. [7], Ibrahim and Yousof [8], Shrivastava et al. [19] and Bhatti et al. [20] considered power LDs and explore the properties of a new generalized Lomax model. Just recently, El-Sherpieny et al. [7] suggested Type-II hybrid censoring method for parametric estimation of LD without due regard being given to the choice of priors 
and posterior risk (PR) associated with the model. This paper fills this gap in the literature and derived the new LD model with minimum PR for the selection of priors.

In this paper, we derive a closed form expression for Bayes estimates (BEs) and PRs using square error loss function (SELF), weighted loss function (WLF), QLF and DeGroot loss function (DLF). Prior predictive approach is used to elicit the hyperparameters of mixture model. We consider Bayesian estimation of two-component mixture of LD assuming censored data of Type I. Different priors and loss functions are considered for comparison. The rest of the paper is structured as follows. Section 2 introduces the two-component mixture model and constructs its likelihood function. In Section 3, posterior distribution, Bayes estimator (BE) and their PRs are derived. In Section 4, uninformative and informative priors are given. Elicitation of hyperparameters is presented in Section 5. Simulation study is conducted in Section 6 along with an application using real-life data and some concluding remarks are given in the final Section 7.

\section{THE TWO-COMPONENT MIXTURE MODEL}

The mixture distribution function with two components of LD having unknown population parameters $\theta_{1}$ and $\theta_{2}$ with unknown mixing weights ( $p$ and $q$, where $q=1-p)$ is

$$
F(x)=p F_{1}(x)+(1-p) F_{2}(x) \quad 0<p<1
$$

where

$$
F_{i}(x)=1-(x+1)^{-\theta_{i}} \quad 0<x<\infty
$$

Thus

$$
F(x)=p\left(1-(x+1)^{-\theta_{1}}\right)+(1-p)\left(1-(x+1)^{-\theta_{2}}\right) \quad i=1,2, \theta_{i}>0
$$

The corresponding mixture density function is

$$
f(x)=p f_{1}(x)+(1-p) f_{2}(x) \quad 0<p<1
$$

The following LD is assumed for both components of mixture with scale parameter $\lambda=1$.

$$
f_{i}(x)=\theta_{i}(1+x)^{-\left(\theta_{i}+1\right)} \quad i=1,2, \theta_{i}>0,0<x<\infty
$$

Thus, the mixture model becomes

$$
\begin{aligned}
& f(x)=p \theta_{1}(x+1)^{-\left(\theta_{1}+1\right)}+(1-p) \theta_{2}(x+1)^{-\left(\theta_{2}+1\right)} \\
& =p \frac{\theta_{1}}{(x+1)} e^{-\theta_{1} \ln (x+1)}+(1-p) \frac{\theta_{2}}{(x+1)} e^{-\theta_{2} \ln (x+1)} \quad, 0<p<1
\end{aligned}
$$

\subsection{The Likelihood Function}

Suppose for testing reliability of units, a random sample of size $n\left(x_{1}, x_{2}, x_{3}, \ldots, x_{n}\right)$ is selected. Let $r$ units in the sample have lifetime in $\left[0, t_{0}\right]$ where $t_{0}$ is a fixed termination time for the experiment and the rest of $n-r$ units survive after the termination time is over. Consider the same situation for sampling from each of the two subpopulations of the model, i.e., from $n_{i}$ units $r_{i}$ have lifetime only in the interval $\left[0, t_{0}\right]$ and $n_{i}-r_{i}$ units survive even after the termination time for $i=1,2$ such that $r=r_{1}+r_{2}$ are the uncensored units. Suppose $x_{i j}$ be the failure time of the $j^{\text {th }}$ unit associated with $i^{\text {th }}$ sub population where $i=1,2, j=1,2, \ldots, r_{i}, 0<x_{1 j}, x_{2 j} \leq t_{0}$. For the above conditions, the Likelihood function is defined by Mendenhall and Hader [21] as

$$
L\left(\theta_{1}, \theta_{2}, p\right) \propto\left\{\prod_{j=1}^{r_{1}} p f_{1}\left(\mathbf{x}_{1 j}\right)\right\}\left\{\prod_{j=1}^{r_{2}}(1-p) f_{2}\left(\mathbf{x}_{2 j}\right)\right\}\left\{1-F\left(t_{0}\right)\right\}^{n-r}
$$

where $\mathbf{x}=\left[\mathbf{x}_{1 j}, \mathbf{x}_{2 j}\right], \mathbf{x}_{1 j}=\left[x_{11}, x_{12}, x_{13}, \ldots, x_{1 r_{1}}\right], \mathbf{x}_{2 j}=\left[x_{21}, x_{22}, x_{23}, \ldots, x_{2 r_{2}}\right]$. 
Substituting the values, we have

$$
\begin{aligned}
L\left(\theta_{1}, \theta_{2}, p\right) \propto & {\left[\prod_{j=1}^{r_{1}} p \frac{\theta_{1}}{\left(\mathbf{x}_{1 j}+1\right)^{\theta_{1}+1}}\right]\left[\prod_{j=1}^{r_{2}} p \frac{\theta_{2}}{\left(\mathbf{x}_{2 j}+1\right)^{\theta_{2}+1}}\right] } \\
& {\left[1-\left\{p\left(1-e^{-\theta_{1} \ln \left(1+t_{0}\right)}\right)+(1-p)\left(1-e^{-\theta_{2} \ln \left(1+t_{0}\right)}\right)\right\}\right]^{n-r} }
\end{aligned}
$$

Now

$$
\begin{aligned}
{\left[1-F\left(t_{0}\right)\right]^{n-r} } & =\left[1-\left\{p\left(1-e^{-\theta_{1} \ln \left(1+t_{0}\right)}\right)+(1-p)\left(1-e^{-\theta_{2} \ln \left(1+t_{0}\right)}\right)\right\}\right]^{n-r} \\
& =\sum_{m=0}^{n-r}\left(\begin{array}{c}
n-r \\
m
\end{array}\right)(1-p)^{m} e^{-\theta_{2} m \ln \left(1+t_{0}\right)} p^{n-r-m} e^{-\theta_{1}(n-r-m) \ln \left(1+t_{0}\right)}
\end{aligned}
$$

Thus, the Likelihood function becomes

$$
\begin{aligned}
L\left(\theta_{1}, \theta_{2}, p\right) \propto & \sum_{m=0}^{n-r}\left(\begin{array}{c}
n-r \\
m
\end{array}\right) p^{n-r_{2}-m}(1-p)^{m+r_{2}} \theta_{1}^{r_{1}} \theta_{2}^{r_{2}} \\
& e^{-\theta_{1}\left[\sum_{j=1}^{r_{1}} \ln \left(x_{1 j}+1\right)+(n-r-m) \ln \left(1+t_{0}\right)\right]_{e}-\theta_{2}\left[\sum_{j=1}^{r_{2}} \ln \left(x_{2 j}+1\right)+(m) \ln \left(1+t_{0}\right)\right]}
\end{aligned}
$$

In next section we will obtain uniform and Jeffreys priors which are the most common examples of uninformative priors which are specified when parameters are bounded.

\section{BAYESIAN ESTIMATION USING UNIFORM PRIOR}

Let $\theta_{1} \theta_{2} \sim U(0, \infty)$ and $p \sim U(0,1)$, where $U$ stand for independent uniform distributions. Assuming independence, we incorporate the joint prior using likelihood function (10) to obtain the joint posterior distribution as in (11) below:

$$
p\left(\theta_{1}, \theta_{2}, p \mid \mathbf{x}\right)=\frac{\sum_{m=0}^{n-r}\left(\begin{array}{c}
n-r \\
m
\end{array}\right) p^{n-r_{2}-m}(1-p)^{m+r_{2}} \theta_{1}^{r_{1}} \theta_{2}^{r_{2}} e^{-\theta_{1} W} e^{-\theta_{2} X}}{A}
$$

where in (11), $\theta_{1}, \theta_{2}>0,0<p<1, W=\sum_{j=1}^{r_{1}} \ln \left(x_{1 j}+1\right)+(n-r-m) \ln \left(1+t_{0}\right)$,

$X=\sum_{j=1}^{r_{2}} \ln \left(x_{2 j}+1\right)+(m) \ln \left(1+t_{0}\right)$ and the denominator of $(11)$ is

$$
A=\sum_{m=0}^{n-r}\left(\begin{array}{c}
n-r \\
m
\end{array}\right) \frac{\Gamma\left(n-r_{2}-m+1\right) \Gamma\left(m+r_{2}+1\right) \Gamma\left(r_{1}+1\right) \Gamma\left(r_{2}+1\right)}{\Gamma(n+2)\left[\sum_{j=1}^{r_{1}} \ln \left(x_{1 j}+1\right)+(n-r-m) \ln \left(1+t_{0}\right)\right]^{r_{1}+1}\left[\sum_{j=1}^{r_{2}} \ln \left(x_{2 j}+1\right)+(m) \ln \left(1+t_{0}\right)\right]^{r_{2}+1}}
$$

The marginal posterior distribution of each parameter is obtained by integrating out the nuisance parameters. The marginal posterior distributions for $\theta_{1}, \theta_{2}, p$ are given in (12), (13) and (14), respectively.

$$
\frac{\sum_{m=0}^{n-r}\left(\begin{array}{c}
n-r \\
m
\end{array}\right) \theta_{1}^{r_{1}} e^{-\theta_{1}\left[\sum_{j=1}^{r_{1}} \ln \left(x_{1 j}+1\right)+(n-r-m) \ln \left(1+t_{0}\right)\right]} \frac{\Gamma\left(n-r_{2}-m+1\right) \Gamma\left(m+r_{2}+1\right) \Gamma\left(r_{2}+1\right)}{\Gamma(n+2)\left[\sum_{j=1}^{r_{2}} \ln \left(x_{2 j}+1\right)+(m) \ln \left(1+t_{0}\right)\right]^{r_{2}+1}}}{A}
$$




$$
\begin{aligned}
& =\frac{\sum_{m=0}^{n-r}\left(\begin{array}{c}
n-r \\
m
\end{array}\right) \theta_{2}^{r_{2}} e^{-\theta_{2}\left[\sum_{j=1}^{r_{1}} \ln \left(x_{2 j}+1\right)+(m) \ln \left(1+t_{0}\right)\right]} \frac{\Gamma\left(n-r_{2}-m+1\right) \Gamma\left(m+r_{2}+1\right) \Gamma\left(r_{1}+1\right)}{\Gamma(n+2)\left[\sum_{j=1}^{r_{2}} \ln \left(x_{1 j}+1\right)+(n-r-m) \ln \left(1+t_{0}\right)\right]^{r_{1}+1}}}{A} \\
& g\left(\theta_{2} \mid \mathbf{x}\right)=
\end{aligned}
$$

\section{1. $B E$ and PR}

Bayesian estimation relates to a loss function, specifying the loss which is obtained when the estimate deviate from the true parameter. A function of the deviation of observed event from the corresponding true event is known as loss function and it represents the cost associated with the event. Loss functions have a wide range of application in Bayesian statistics. It is basically used for parameter estimation and prediction. In order to obtain best estimator, the loss criterion is specified in decision theory. SELF is the simplest form of symmetric loss function. SELF was introduced by Legendre [22] and Gauss [23] to develop least square theory. It gives equal importance to both positive and negative errors. But this assumption may not be suitable in all estimation problems because overestimation may cause serious results. Thus, use of asymmetric loss functions is suggested. Norstrom [24] suggested an asymmetric recautionary loss function (PLF) and introduced a general class of PLF with QLF as its special case. WLF is generalization of SELF. DeGroot also discussed an asymmetric loss function. In this paper, BEs are estimated under SELF, QLF, WLF, PLF and DLF. Table 1 shows BEs and PRs for above said loss functions.

Using the marginal posterior distributions of $\theta_{1}, \theta_{2}, p$, we derive the BEs and PRs under uniform prior using SELF. The BEs of $\theta_{1}, \theta_{2}, p$ are given in (15), (16) and (17), respectively.

$$
=\frac{\sum_{m=0}^{n-r}\left(\begin{array}{c}
n-r \\
m
\end{array}\right) \frac{\Gamma\left(n-r_{2}-m+1\right) \Gamma\left(m+r_{2}+1\right) \Gamma\left(r_{1}+2\right) \Gamma\left(r_{2}+1\right)}{\Gamma(n+2)\left[\sum_{j=1}^{r_{1}} \ln \left(x_{1 j}+1\right)+(n-r-m) \ln \left(1+t_{0}\right)\right]^{r_{1}+2}\left[\sum_{j=1}^{r_{2}} \ln \left(x_{2 j}+1\right)+(m) \ln \left(1+t_{0}\right)\right]^{r_{2}+1}}}{A}
$$

Table 1 BE and PR under different loss functions.

\begin{tabular}{lll}
\hline Loss Function & Bayes Estimator (BE) & Posterior Risk (PR) \\
\hline SELF $=\left(\theta-\theta^{*}\right)^{2}$ & $E_{\theta \mid x}(\theta)$ & $E_{\theta \mid x}\left(\theta^{2}\right)-\left[E_{\theta \mid x}(\theta)\right]^{2}$ \\
$\mathrm{WLF}=\frac{\left(\theta-\theta^{*}\right)^{2}}{\theta}$ & {$\left[E_{\theta \mid x}\left(\theta^{-1}\right)\right]^{-1}$} & $E_{\theta \mid x}(\theta)-\left[E_{\theta \mid x}\left(\theta^{-1}\right)\right]^{-1}$ \\
$\mathrm{QLF}=\frac{\left(\theta-\theta^{*}\right)^{2}}{\theta^{2}}$ & $\frac{E_{\theta \mid x}\left(\theta^{-1}\right)}{E_{\theta \mid x}\left(\theta^{-2}\right)}$ & $1-\frac{\left[E_{\theta \mid x}\left(\theta^{-1}\right)\right]^{2}}{E_{\theta \mid x}\left(\theta^{-2}\right)}$ \\
$\mathrm{PLF}=\frac{\left(\theta-\theta^{*}\right)^{2}}{\theta^{*}}$ & $\sqrt{E_{\theta \mid x}\left(\theta^{2}\right)}$ & $2\left[\sqrt{E_{\theta \mid x}\left(\theta^{2}\right)}-E_{\theta \mid x}(\theta)\right]$ \\
$\mathrm{DLF}=\left(\frac{\theta-\theta^{*}}{\theta^{*}}\right)^{2}$ & $\frac{E_{\theta \mid x}\left(\theta^{2}\right)}{E_{\theta \mid x}(\theta)}$ & $1-\frac{\left[E_{\theta \mid x}(\theta)\right]^{2}}{E_{\theta \mid x}\left(\theta^{2}\right)}$ \\
\hline
\end{tabular}


Similarly, BEs using WLF, QLF, DLF and PLF can be derived.

The PRs of $\theta_{1}, \theta_{2}, p$ are

$$
=\frac{\sum_{m=0}^{n-r}\left(\begin{array}{c}
n-r \\
m
\end{array}\right) \frac{\Gamma\left(n-r_{2}-m+1\right) \Gamma\left(m+r_{2}+1\right) \Gamma\left(r_{1}+3\right) \Gamma\left(r_{2}+1\right)}{\Gamma(n+2)\left[\sum_{j=1}^{r_{1}} \ln \left(x_{1 j}+1\right)+(n-r-m) \ln \left(1+t_{0}\right)\right]^{r_{1}+3}\left[\sum_{j=1}^{r_{2}} \ln \left(x_{2 j}+1\right)+(m) \ln \left(1+t_{0}\right)\right]^{r_{2}+1}}}{A}-\left(\theta_{1}\right)^{2}
$$

$$
\rho\left(\theta_{2}\right)=\frac{\sum_{m=0}^{n-r}\left(\begin{array}{c}
n-r \\
m
\end{array}\right) \frac{\Gamma\left(n-r_{2}-m+1\right) \Gamma\left(m+r_{2}+1\right) \Gamma\left(r_{1}+1\right) \Gamma\left(r_{2}+3\right)}{\Gamma(n+2)\left[\sum_{j=1}^{r_{1}} \ln \left(x_{1 j}+1\right)+(n-r-m) \ln \left(1+t_{0}\right)\right]^{r_{1}+1}\left[\sum_{j=1}^{r_{2}} \ln \left(x_{2 j}+1\right)+(m) \ln \left(1+t_{0}\right)\right]^{r_{2}+3}}-\left(\theta_{2}\right)^{2}}{A}
$$

$$
\frac{\sum_{m=0}^{n-r}\left(\begin{array}{c}
n-r \\
m
\end{array}\right) \frac{\Gamma\left(n-r_{2}-m+3\right) \Gamma\left(m+r_{2}+1\right) \Gamma\left(r_{1}+1\right) \Gamma\left(r_{2}+1\right)}{\Gamma(n+4)\left[\sum_{j=1}^{r_{1}} \ln \left(x_{1 j}+1\right)+(n-r-m) \ln \left(1+t_{0}\right)\right]^{r_{1}+1}\left[\sum_{j=1}^{r_{2}} \ln \left(x_{2 j}+1\right)+(m) \ln \left(1+t_{0}\right)\right]^{r_{2}+1}}}{A}-(p)^{2}
$$

where $\theta_{1}, \theta_{2}$ and $p$ are obtained from (15), (16) and (17), respectively. PRs using WLF, QLF, DLF and PLF can be derived in similar way.

\subsection{Bayesian Estimation Using Jeffrey Prior}

Jeffrey's prior is proportional to the under root of the Fisher's information, i.e., $p(\theta) \propto \sqrt{I(\theta)}$, where $I(\theta)=-E\left(\frac{\partial^{2} \ln L(.)}{\partial \theta^{2}}\right)$ and if $\theta$ is a vector of parameters then Jeffrey's prior is proportional to the determinant of Fisher's Information matrix. Let the Jeffrey's priors for the unknown parameters $\theta_{1}$ and $\theta_{2}$ are $p\left(\theta_{1}\right) \propto \frac{1}{\theta_{1}}, p\left(\theta_{2}\right) \propto \frac{1}{\theta_{2}}$ and $p(p)=1$ for $\theta_{1}, \theta_{2}>0$ and $0<p<1$. Assuming independence, we incorporate the joint prior $p\left(\theta_{1}, \theta_{2}, p \mid x\right) \propto \frac{1}{\theta_{1} \theta_{2}}$ with likelihood function $(\log )$ to get the joint posterior distribution of $\theta_{1}, \theta_{2}$ and $p$ as

$$
p\left(\theta_{1}, \theta_{2}, p \mid \mathbf{x}\right)=\frac{\sum_{m=0}^{n-r}\left(\begin{array}{c}
n-r \\
m
\end{array}\right) p^{n-r_{2}-m}(1-p)^{m+r_{2}} \theta_{1}^{r_{1}-1} \theta_{2}^{r_{2}-1} e^{-\theta_{1} Q} e^{-\theta_{2} Z}}{B}
$$

where

$$
\begin{aligned}
& \theta_{1,} \theta_{2}>0,0<p<1, Q=\sum_{j=1}^{r_{1}} \ln \left(x_{1 j}+1\right)+(n-r-m) \ln \left(1+t_{0}\right), \\
& Z=\sum_{j=1}^{r_{2}} \ln \left(x_{2 j}+1\right)+(m) \ln \left(1+t_{0}\right)
\end{aligned}
$$

and

$$
B=\sum_{m=0}^{n-r}\left(\begin{array}{c}
n-r \\
m
\end{array}\right) \frac{\Gamma\left(n-r_{2}-m+1\right) \Gamma\left(m+r_{2}+1\right) \Gamma\left(r_{1}\right) \Gamma\left(r_{2}\right)}{\Gamma(n+2)\left[\sum_{j=1}^{r_{1}} \ln \left(x_{1 j}+1\right)+(n-r-m) \ln \left(1+t_{0}\right)\right]^{r_{1}}\left[\sum_{j=1}^{r_{2}} \ln \left(x_{2 j}+1\right)+(m) \ln \left(1+t_{0}\right)\right]^{r_{2}}} .
$$

The marginal posterior distribution of each parameter is obtained by integrating out the nuisance parameters. 


\subsection{BE and PR}

Using the marginal posterior distributions of $\theta_{1}, \theta_{2}, p$, we derive the BEs and PRs under Jeffrey's prior using WLF. The BEs of $\theta_{1}, \theta_{2}, p$ are given.

$$
\begin{gathered}
\theta_{1}=\frac{B}{\sum_{m=0}^{n-r}\left(\begin{array}{c}
n-r \\
m
\end{array}\right) \frac{\Gamma\left(n-r_{2}-m+1\right) \Gamma\left(m+r_{2}+1\right) \Gamma\left(r_{1}-1\right) \Gamma\left(r_{2}\right)}{\Gamma(n+2)\left[\sum_{j=1}^{r_{1}} \ln \left(x_{1 j}+1\right)+(n-r-m) \ln \left(1+t_{0}\right)\right]^{r_{1}-1}\left[\sum_{j=1}^{r_{2}} \ln \left(x_{2 j}+1\right)+(m) \ln \left(1+t_{0}\right)\right]^{r_{2}}}} \\
\theta_{2}=\frac{B}{\sum_{m=0}^{n-r}\left(\begin{array}{c}
n-r \\
m
\end{array}\right) \frac{\Gamma\left(n-r_{2}-m+1\right) \Gamma\left(m+r_{2}+1\right) \Gamma\left(r_{1}\right) \Gamma\left(r_{2}-1\right)}{\Gamma(n+2)\left[\sum_{j=1}^{r_{1}} \ln \left(x_{1 j}+1\right)+(n-r-m) \ln \left(1+t_{0}\right)\right]^{r_{1}}\left[\sum_{j=1}^{r_{2}} \ln \left(x_{2 j}+1\right)+(m) \ln \left(1+t_{0}\right)\right]^{r_{2}-1}}} \\
p=\frac{B}{\sum_{m=0}^{n-r}\left(\begin{array}{c}
n-r \\
m
\end{array}\right) \frac{\Gamma\left(n-r_{2}-m\right) \Gamma\left(m+r_{2}+1\right) \Gamma\left(r_{1}\right) \Gamma\left(r_{2}\right)}{\Gamma(n+1)}\left[\sum_{j=1}^{r_{1}} \ln \left(x_{1 j}+1\right)+(n-r-m) \ln \left(1+t_{0}\right)\right]^{r_{1}}\left[\sum_{j=1}^{r_{2}} \ln \left(x_{2 j}+1\right)+(m) \ln \left(1+t_{0}\right)\right]^{r_{2}}}
\end{gathered}
$$

The PRs of $\theta_{1}, \theta_{2}, p$ under Jeffrey's prior using WLF are

$$
\begin{aligned}
& \rho\left(\theta_{1}\right)=\frac{\sum_{m=0}^{n-r}\left(\begin{array}{c}
n-r \\
m
\end{array}\right) \frac{\Gamma\left(n-r_{2}-m+1\right) \Gamma\left(m+r_{2}+1\right) \Gamma\left(r_{1}+1\right) \Gamma\left(r_{2}\right)}{\Gamma(n+2)\left[\sum_{j=1}^{r_{1}} \ln \left(x_{1 j}+1\right)+(n-r-m) \ln \left(1+t_{0}\right)\right]^{r_{1}+1}\left[\sum_{j=1}^{r_{2}} \ln \left(x_{2 j}+1\right)+(m) \ln \left(1+t_{0}\right)\right]^{r_{2}}}-\theta_{1}}{B} \\
& \rho\left(\theta_{2}\right)=\frac{\sum_{m=0}^{n-r}\left(\begin{array}{c}
n-r \\
m
\end{array}\right) \frac{\Gamma\left(n-r_{2}-m+1\right) \Gamma\left(m+r_{2}+1\right) \Gamma\left(r_{1}\right) \Gamma\left(r_{2}+1\right)}{\Gamma(n+2)\left[\sum_{j=1}^{r_{1}} \ln \left(x_{1 j}+1\right)+(n-r-m) \ln \left(1+t_{0}\right)\right]^{r_{1}}\left[\sum_{j=1}^{r_{2}} \ln \left(x_{2 j}+1\right)+(m) \ln \left(1+t_{0}\right)\right]^{r_{2}+1}}-\theta_{2}}{B} \\
& \rho(p)=\frac{\sum_{m=0}^{n-r}\left(\begin{array}{c}
n-r \\
m
\end{array}\right) \frac{\Gamma\left(n-r_{2}-m+2\right) \Gamma\left(m+r_{2}+1\right) \Gamma\left(r_{1}\right) \Gamma\left(r_{2}\right)}{\Gamma(n+3)\left[\sum_{j=1}^{r_{1}} \ln \left(x_{1 j}+1\right)+(n-r-m) \ln \left(1+t_{0}\right)\right]^{r_{1}}\left[\sum_{j=1}^{r_{2}} \ln \left(x_{2 j}+1\right)+(m) \ln \left(1+t_{0}\right)\right]^{r_{2}}}-p}{B}-p
\end{aligned}
$$

where $\theta_{1}, \theta_{2}$ and $p$ are obtained from (22), (23) and (24), respectively. BEs and PRs using SELF, QLF, DLF and PLF can be derived in similar way.

\subsection{Informative Priors}

When the functional form of prior density is assumed to be known, the density is known as subjective prior or informative prior. Thus, the previous scientific knowledge can be used in statistical inference by manipulating prior information to have a posterior distribution. Informative prior provides more information and the analysis are more accurate, so they are preferred over noninformative priors. 


\section{BAYESIAN ESTIMATION USING GAMMA PRIOR}

Let $\theta_{1} \sim \operatorname{Gamma}(a, b), \theta_{2} \sim \operatorname{Gamma}(c, d)$ and $p \sim \operatorname{Beta}(e, f)$ distribution with the assumption of independence, then the joint prior distribution of $\theta_{1}, \theta_{2}$ and $p$ is

$$
p\left(\theta_{1}, \theta_{2}, p\right) \propto \theta_{1}^{a-1} \theta_{2}^{c-1} p^{e-1}(1-p)^{f-1} e^{-b \theta_{1}} e^{-d \theta_{2}} \quad \theta_{1}, \theta_{2}>0,0<p<1
$$

By incorporating the joint prior with the likelihood function $(\log )$, the joint posterior distribution of $\theta_{1}, \theta_{2}$ and $p$ is

$$
p\left(\theta_{1}, \theta_{2}, p \mid \mathbf{x}\right)=\frac{\sum_{m=0}^{n-r}\left(\begin{array}{c}
n-r \\
m
\end{array}\right) p^{e+n-r_{2}-m-1}(1-p)^{f+m+r_{2}-1} \theta_{1}^{a+r_{1}-1} \theta_{2}^{c+r_{2}-1} e^{-\theta_{1} g_{e} e^{-\theta_{2} k}}}{H}
$$

where

$$
\begin{aligned}
& \theta_{1}, \theta_{2}>0,0<p<1, g=\left[b+\sum_{j=1}^{r_{1}} \ln \left(x_{1 j}+1\right)+(n-r-m) \ln \left(1+t_{0}\right)\right], \\
& k=\left[d+\sum_{j=1}^{r_{2}} \ln \left(x_{2 j}+1\right)+(m) \ln \left(1+t_{0}\right)\right]
\end{aligned}
$$

and

$$
H=\sum_{m=0}^{n-r}\left(\begin{array}{c}
n-r \\
m
\end{array}\right) \frac{\Gamma\left(n+e-r_{2}-m\right) \Gamma\left(f+m+r_{2}\right) \Gamma\left(a+r_{1}\right) \Gamma\left(c+r_{2}\right)}{\Gamma(e+f+n)\left[b+\sum_{j=1}^{r_{1}} \ln \left(x_{1 j}+1\right)+(n-r-m) \ln \left(1+t_{0}\right)\right]^{a+r_{1}}\left[d+\sum_{j=1}^{r_{2}} \ln \left(x_{2 j}+1\right)+(m) \ln \left(1+t_{0}\right)\right]^{c+r_{2}}} .
$$

The marginal posterior distribution of each parameter is obtained by integrating out the nuisance parameters.

\section{1. $B E$ and PR}

As in previous section, we can use the marginal posterior distributions of $\theta_{1}, \theta_{2}, p$, and can derive the BEs and PRs under Gamma prior using PLF. The BEs of $\theta_{1}, \theta_{2}, p$ are

$$
\theta_{1}=\sqrt{\frac{\sum_{m=0}^{n-r}\left(\begin{array}{c}
n-r \\
m
\end{array}\right) \frac{\Gamma\left(n+e-r_{2}-m\right) \Gamma\left(f+m+r_{2}\right) \Gamma\left(a+r_{1}+2\right) \Gamma\left(c+r_{2}\right)}{\Gamma(n+e+f)}\left[b+\sum_{j=1}^{r_{1}} \ln \left(x_{1 j}+1\right)+(n-r-m) \ln \left(1+t_{0}\right)\right]^{a+r_{1}+2}\left[d+\sum_{j=1}^{r_{2}} \ln \left(x_{2 j}+1\right)+(m) \ln \left(1+t_{0}\right)\right]^{c+r_{2}}}{H}}
$$

$$
\theta_{2}=\sqrt{\frac{\sum_{m=0}^{n-r}\left(\begin{array}{c}
n-r \\
m
\end{array}\right) \frac{\Gamma\left(n+e-r_{2}-m\right) \Gamma\left(f+m+r_{2}\right) \Gamma\left(a+r_{1}\right) \Gamma\left(c+r_{2}+2\right)}{\Gamma(n+e+f)\left[b+\sum_{j=1}^{r_{1}} \ln \left(x_{1 j}+1\right)+(n-r-m) \ln \left(1+t_{0}\right)\right]^{a+r_{1}}\left[d+\sum_{j=1}^{r_{2}} \ln \left(x_{2 j}+1\right)+(m) \ln \left(1+t_{0}\right)\right]^{c+r_{2}+2}}}{H}}
$$

$$
p=\sqrt{\frac{\sum_{m=0}^{n-r}\left(\begin{array}{c}
n-r \\
m
\end{array}\right) \frac{\Gamma\left(n+e-r_{2}-m+2\right) \Gamma\left(f+m+r_{2}\right) \Gamma\left(a+r_{1}\right) \Gamma\left(c+r_{2}\right)}{\Gamma(n+e+f+2)\left[b+\sum_{j=1}^{r_{1}} \ln \left(x_{1 j}+1\right)+(n-r-m) \ln \left(1+t_{0}\right)\right]^{a+r_{1}}\left[d+\sum_{j=1}^{r_{2}} \ln \left(x_{2 j}+1\right)+(m) \ln \left(1+t_{0}\right)\right]^{c+r_{2}}}}{H}}
$$

The posterior risks of $\theta_{1}, \theta_{2}, p$ under Gamma prior using PLF are

$$
\rho\left(\theta_{1}\right)=2\left(\theta_{1}-\frac{A}{H}\right)
$$


where

$$
\begin{gathered}
A=\sum_{m=0}^{n-r}\left(\begin{array}{c}
n-r \\
m
\end{array}\right) \frac{\Gamma\left(n+e-r_{2}-m\right) \Gamma\left(f+m+r_{2}\right) \Gamma\left(a+r_{1}+1\right) \Gamma\left(c+r_{2}\right)}{\Gamma(n+e+f)\left[b+\sum_{j=1}^{r_{1}} \ln \left(x_{1 j}+1\right)+(n-r-m) \ln \left(1+t_{0}\right)\right]^{a+r_{1}+1}\left[d+\sum_{j=1}^{r_{2}} \ln \left(x_{2 j}+1\right)+(m) \ln \left(1+t_{0}\right)\right]^{c+r_{2}}} . \\
\rho\left(\theta_{2}\right)=2\left(\theta_{2}-\frac{B}{H}\right)
\end{gathered}
$$

where

$$
\begin{gathered}
B=\sum_{m=0}^{n-r}\left(\begin{array}{c}
n-r \\
m
\end{array}\right) \frac{\Gamma\left(n+e-r_{2}-m\right) \Gamma\left(f+m+r_{2}\right) \Gamma\left(a+r_{1}\right) \Gamma\left(c+r_{2}+1\right)}{\Gamma(n+e+f)\left[b+\sum_{j=1}^{r_{1}} \ln \left(x_{1 j}+1\right)+(n-r-m) \ln \left(1+t_{0}\right)\right]^{a+r_{1}}\left[d+\sum_{j=1}^{r_{2}} \ln \left(x_{2 j}+1\right)+(m) \ln \left(1+t_{0}\right)\right]^{c+r_{2}+1}} . \\
\rho(p)=2\left(p-\frac{C}{H}\right)
\end{gathered}
$$

where

$$
C=\sum_{m=0}^{n-r}\left(\begin{array}{c}
n-r \\
m
\end{array}\right) \frac{\Gamma\left(n+e-r_{2}-m+1\right) \Gamma\left(f+m+r_{2}\right) \Gamma\left(a+r_{1}\right) \Gamma\left(c+r_{2}\right)}{\Gamma(n+e+f+1)\left[b+\sum_{j=1}^{r_{1}} \ln \left(x_{1 j}+1\right)+(n-r-m) \ln \left(1+t_{0}\right)\right]^{a+r_{1}}\left[d+\sum_{j=1}^{r_{2}} \ln \left(x_{2 j}+1\right)+(m) \ln \left(1+t_{0}\right)\right]^{c+r_{2}} .}
$$

where $\theta_{1}, \theta_{2}$ and $p$ are obtained from (27-29), respectively. BEs and PRs using SELF, QLF, WLF and DLF can be derived in similar way.

\subsection{Bayesian Estimation Using Inverse Levy Prior}

Let $\theta_{1} \sim \operatorname{InverseLevy}(a), \theta_{2} \sim \operatorname{InverseLevy}(c)$ and $p \sim \operatorname{Beta}(e, f)$ so the joint prior distribution of $\theta_{1}, \theta_{2}$ and $p$ is

$$
p\left(\theta_{1}, \theta_{2}, p\right) \propto \theta_{1}^{-\frac{1}{2}} \theta_{2}^{-\frac{1}{2}} p^{e-1}(1-p)^{f-1} e^{-\frac{a \theta_{1}}{2}} e^{-\frac{c \theta_{2}}{2}} \quad \theta_{1}, \theta_{2}>0,0<p<1
$$

Using above joint prior and the likelihood function (10), the joint posterior distribution of $\theta_{1,}, \theta_{2}$ and $p$ is

$$
p\left(\theta_{1}, \theta_{2}, p \mid \mathbf{x}\right)=\frac{\sum_{m=0}^{n-r}\left(\begin{array}{c}
n-r \\
m
\end{array}\right) p^{e+n-r_{2}-m-1}(1-p)^{f+m+r_{2}-1} \theta_{1}^{r_{1}-1 / 2} \theta_{2}^{r_{2}-1 / 2} e^{-\theta_{1} u} e^{-\theta_{2} v}}{J}
$$

where

$$
\begin{aligned}
& \theta_{1}, \theta_{2}>0,0<p<1, u=\left[\frac{a}{2}+\sum_{j=1}^{r_{1}} \ln \left(x_{1 j}+1\right)+(n-r-m) \ln \left(1+t_{0}\right)\right], \\
& v=\left[\frac{c}{2}+\sum_{j=1}^{r_{2}} \ln \left(x_{2 j}+1\right)+(m) \ln \left(1+t_{0}\right)\right]
\end{aligned}
$$

and

$$
J=\sum_{m=0}^{n-r}\left(\begin{array}{c}
n-r \\
m
\end{array}\right) \frac{\Gamma\left(n+e-r_{2}-m\right) \Gamma\left(f+m+r_{2}\right) \Gamma\left(r_{1}+\frac{1}{2}\right) \Gamma\left(r_{2}+\frac{1}{2}\right)}{\Gamma(n+e+f)\left[\frac{a}{2}+\sum_{j=1}^{r_{1}} \ln \left(x_{1 j}+1\right)+(n-r-m) \ln \left(1+t_{0}\right)\right]^{r_{1}+1 / 2}\left[\frac{c}{2}+\sum_{j=1}^{r_{2}} \ln \left(x_{2 j}+1\right)+(m) \ln \left(1+t_{0}\right)\right]^{r_{2}+1 / 2}} .
$$

The marginal posterior distribution of each parameter is obtained by integrating out the nuisance parameters. 


\subsection{BE and PR}

Using the marginal posterior distributions of $\theta_{1}, \theta_{2}, p$, we derive the BEs and PRs for $\theta_{1}, \theta_{2}, p$ under Inverse Levy prior using DLF as

$$
\hat{\theta}_{1}=\frac{K}{L}, p\left(\hat{\theta}_{1}\right)=1-\left(\frac{L^{2}}{K J}\right)
$$

where

$$
K=\sum_{m=0}^{n-r}\left(\begin{array}{c}
n-r \\
m
\end{array}\right) \frac{\Gamma\left(n+e-r_{2}-m\right) \Gamma\left(f+m+r_{2}\right) \Gamma\left(r_{1}+5 / 2\right) \Gamma\left(r_{2}+1 / 2\right)}{\Gamma(n+e+f)\left[\frac{a}{2}+\sum_{j=1}^{r_{1}} \ln \left(x_{1 j}+1\right)+(n-r-m) \ln \left(1+t_{0}\right)\right]^{r_{1}+5 / 2}\left[\frac{c}{2}+\sum_{j=1}^{r_{2}} \ln \left(x_{2 j}+1\right)+(m) \ln \left(1+t_{0}\right)\right]^{r_{2}+1 / 2}}
$$

and

$$
\begin{gathered}
L=\sum_{m=0}^{n-r}\left(\begin{array}{c}
n-r \\
m
\end{array}\right) \frac{\Gamma\left(n+e-r_{2}-m\right) \Gamma\left(f+m+r_{2}\right) \Gamma\left(r_{1}+3 / 2\right) \Gamma\left(r_{2}+1 / 2\right)}{\Gamma(n+e+f)\left[\frac{a}{2}+\sum_{j=1}^{r_{1}} \ln \left(x_{1 j}+1\right)+(n-r-m) \ln \left(1+t_{0}\right)\right]^{r_{1}+3 / 2}\left[\frac{c}{2}+\sum_{j=1}^{r_{2}} \ln \left(x_{2 j}+1\right)+(m) \ln \left(1+t_{0}\right)\right]^{r_{2}+1 / 2}} . \\
\hat{\theta}_{2}=\frac{M}{N}, p\left(\hat{\theta}_{2}\right)=1-\left(\frac{N^{2}}{M J}\right)
\end{gathered}
$$

where

$$
M=\sum_{m=0}^{n-r}\left(\begin{array}{c}
n-r \\
m
\end{array}\right) \frac{\Gamma\left(n+e-r_{2}-m\right) \Gamma\left(f+m+r_{2}\right) \Gamma\left(r_{1}+1 / 2\right) \Gamma\left(r_{2}+5 / 2\right)}{\Gamma(n+e+f)\left[\frac{a}{2}+\sum_{j=1}^{r_{1}} \ln \left(x_{1 j}+1\right)+(n-r-m) \ln \left(1+t_{0}\right)\right]^{r_{1}+1 / 2}\left[\frac{c}{2}+\sum_{j=1}^{r_{2}} \ln \left(x_{2 j}+1\right)+(m) \ln \left(1+t_{0}\right)\right]^{r_{2}+5 / 2}}
$$

and

$$
\begin{gathered}
N=\sum_{m=0}^{n-r}\left(\begin{array}{c}
n-r \\
m
\end{array}\right) \frac{\Gamma\left(n+e-r_{2}-m\right) \Gamma\left(f+m+r_{2}\right) \Gamma\left(r_{1}+1 / 2\right) \Gamma\left(r_{2}+3 / 2\right)}{\Gamma(n+e+f)\left[\frac{a}{2}+\sum_{j=1}^{r_{1}} \ln \left(x_{1 j}+1\right)+(n-r-m) \ln \left(1+t_{0}\right)\right]^{r_{1}+1 / 2}\left[\frac{c}{2}+\sum_{j=1}^{r_{2}} \ln \left(x_{2 j}+1\right)+(m) \ln \left(1+t_{0}\right)\right]^{r_{2}+3 / 2}} . \\
\hat{p}=\frac{R}{Q}, p(\hat{p})=1-\left(\frac{Q^{2}}{R J}\right)
\end{gathered}
$$

where

$$
R=\sum_{m=0}^{n-r}\left(\begin{array}{c}
n-r \\
m
\end{array}\right) \frac{\Gamma\left(n+e-r_{2}-m+2\right) \Gamma\left(f+m+r_{2}\right) \Gamma\left(r_{1}+1 / 2\right) \Gamma\left(r_{2}+1 / 2\right)}{\Gamma(n+e+f+2)\left[\frac{a}{2}+\sum_{j=1}^{r_{1}} \ln \left(x_{1 j}+1\right)+(n-r-m) \ln \left(1+t_{0}\right)\right]^{r_{1}+1 / 2}\left[\frac{c}{2}+\sum_{j=1}^{r_{2}} \ln \left(x_{2 j}+1\right)+(m) \ln \left(1+t_{0}\right)\right]^{r_{2}+1 / 2}}
$$

and

$$
Q=\sum_{m=0}^{n-r}\left(\begin{array}{c}
n-r \\
m
\end{array}\right) \frac{\Gamma\left(n+e-r_{2}-m+1\right) \Gamma\left(f+m+r_{2}\right) \Gamma\left(r_{1}+1 / 2\right) \Gamma\left(r_{2}+1 / 2\right)}{\Gamma(n+e+f+1)\left[\frac{a}{2}+\sum_{j=1}^{r_{1}} \ln \left(x_{1 j}+1\right)+(n-r-m) \ln \left(1+t_{0}\right)\right]^{r_{1}+1 / 2}\left[\frac{c}{2}+\sum_{j=1}^{r_{2}} \ln \left(x_{2 j}+1\right)+(m) \ln \left(1+t_{0}\right)\right]^{r_{2}+1 / 2}} .
$$

BE and PRs using SELF, WLF, QLF and PLF can be derived in similar way. 


\section{ELICITATION OF HYPERPARAMETERS}

The process of extracting expert knowledge about some unknown quantity of interest or probability of some future event is known as elicitation. It is a process in which personal beliefs are formulated into probability distribution. Aslam [2] proposed some new methods to elicit the hyperparameters of the prior density. Method of elicitation using prior predictive probabilities defined by Aslam [2] is used here to elicit the hyperparameters. Prior predictive distributions using Inverse Levy prior and Gamma prior are obtained by using the following equation:

$$
p(y)=\int_{0}^{\infty} \int_{0}^{\infty} \int_{0}^{1} p\left(\theta_{1}, \theta_{2}, p\right) p\left(y \mid \theta_{1}, \theta_{2}, p\right) d \theta
$$

The prior predictive distribution of using Inverse Levy prior is

$$
p(y)=\frac{1}{2 \sqrt{2}(y+1)(e+f)}\left(\frac{e \sqrt{a}}{\left[\frac{a}{2}+\ln (y+1)\right]^{\frac{3}{2}}}+\frac{f \sqrt{c}}{\left[\frac{c}{2}+\ln (y+1)\right]^{\frac{3}{2}}}\right), \quad y>0
$$

The expert's probabilities for prior predictive distribution using Inverse Levy prior are assumed to be $0.20,0.40,0.45$ and 0.50 associated with intervals $0<y<1,0<y<5,0<y<10$ and $0<y<20$ respectively as

$$
\int_{0}^{1} p(y) d y=0.20, \int_{0}^{5} p(y) d y=0.40, \int_{0}^{10} p(y) d y=0.40, \int_{0}^{20} p(y) d y=0.50
$$

where $p(y)$ is obtained from predictive IL. We consider four intervals to elicit four hyperparameters. Solving the above four equations simultaneously through computer program developed in SAS using "Proc SYSLIN" command gives the elicited values of hyperparameters as $a=0.498211, c=2.000367, e=0.498211$ and $f=1.300001$.

Similarly, the prior predictive distribution of $Y$ using Gamma prior is

$$
p(y)=\frac{1}{(y+1)(e+f)}\left(\frac{a b^{a} e}{[b+\ln (y+1)]^{a+1}}+\frac{c d^{c} f}{[d+\ln (y+1)]^{c+1}}\right), y>0
$$

The expert's probabilities for prior predictive distribution using Gamma prior are assumed to be 0.65, 0.80, 0.85, 0.90, 0.92 and 0.95 associated with intervals $0<y<1,0<y<2,0<y<5,0<y<6,0<y<8$ and $0<y<10$, respectively. Following the procedure used for Inverse Levy prior, the elicited values of hyperparameters of Gamma prior are $a=2.999355, b=0.999900$, $c=3.000051, d=2.000081, e=0.700317$ and $f=1.000200$.

\section{EMPIRICAL STUDY}

This section contains empirical study using simulated data and real-life data for employing LD model. Let's begin with simulation study followed by real-life data in the following subsections.

\subsection{Applications Using Simulated Data}

A simulation work is carried out in order to investigate properties of BEs and the effect of different sample size, censoring rate and mixing proportion using different priors. Samples of size $(n=30,50,100,500,1000)$ having censoring rate $t_{0} \in(2,6)$ are randomly generated from the two-component mixture of LD (having scale parameters equal to unity) with unknown parameter $\theta_{1}, \theta_{2}$ and $p$ such that $\left(\theta_{1}, \theta_{2}\right) \in$ $\{(0.5,1),(1,1),(5,3),(3,3)\}$ and $p \in(0.4,0.7)$.

For generating random data from mixture of Lomax model, we adopted the method of probabilistic mixing method by inverse transformation of distribution function. A random no. $u_{i}$ is generated from the uniform distribution $(0,1)$ and using the relation of inverse distribution function $x_{i}=F^{-1}\left(u_{i}\right)$, we obtain the data for both components of mixture variable. If $u_{i}<p$, the observation was taken randomly from LD with parameter $\theta_{1}$ and if $u_{i}>p$, the observation was taken randomly from LD with parameter $\theta_{2}$. Right censoring of Type I using a fixed termination time $t_{0}$ is done. Two different values for censoring time are chosen to assess the influence of censoring rate on estimates. 
For each of different combinations of parameter, sample size and censoring rate 10000 samples were generated in Mathematica. For each of 10000 samples, BEs and PRs were calculated and average of these is presented in Tables 2-5. Extensive simulation study is conducted for different combinations of $\theta_{1}, \theta_{2}$, i.e., $\theta_{1}<\theta_{2}, \theta_{1}>\theta_{2}$ and $\theta_{1}=\theta_{2}$ for different values of $t_{0}$ and $p$. For the sake of simplicity only some of these results are presented here.

The results declare that $\mathrm{PR}$ of the parameters decreases with the increase in sample size. The unknown population parameter $\theta_{1}, \theta_{2}$ are over estimated and underestimated due to the result of censoring. Mostly $\theta_{1}$ is over estimated and $\theta_{2}$ is underestimated but this discrepancy reduces as we increase the proportion parameter, i.e., with the increase in mixing proportion, the extent of over estimation and under estimation reduces and the estimates becomes more accurate. Also, the PRs show different patterns. Increasing the sample size, PR for all $\theta_{1}, \theta_{2}$ and $p$ decreases but increasing the mixing weight while keeping other things constant, the risk for $\theta_{1}$ and $p$ decreases but for $\theta_{2}$ it increases.

While comparing the uninformative priors, it is observed that the estimates are more over and underestimated for UP thus the estimates for JP are more accurate. Also, in terms of PRs, it is clear that risks using JP are smaller than UP. Among the informative priors, estimates are more accurately estimated for ILP. Also, in terms of PRs, risks using ILP are smaller than the posterior risks using GP. Overall comparison of informative and noninformative priors because of Bayes PR shows that ILP is preferable.

Table 2 BEs and PRs using uniform prior.

\begin{tabular}{llcccccc}
\hline \multirow{2}{*}{$t_{0}=73$} & & $\boldsymbol{\theta}_{\mathbf{1}}$ & $\boldsymbol{p}\left(\boldsymbol{\theta}_{\mathbf{1}}\right)$ & $\boldsymbol{\theta}_{\mathbf{2}}$ & $\boldsymbol{p}\left(\boldsymbol{\theta}_{\mathbf{2}}\right)$ & $\boldsymbol{p}$ & $\boldsymbol{p}(\boldsymbol{p})$ \\
& SELF & 0.198063 & 0.002174 & 0.319121 & 0.035580 & 0.807293 & 0.006614 \\
& QLF & 0.177587 & 0.053427 & 0.144049 & 0.347271 & 0.787792 & 0.012944 \\
& WLF & 0.187610 & 0.010452 & 0.220688 & 0.098432 & 0.798123 & 0.009169 \\
& DLF & 0.209044 & 0.052530 & 0.430616 & 0.258919 & 0.815486 & 0.010047 \\
& PLF & 0.203479 & 0.010833 & 0.370700 & 0.103158 & 0.811379 & 0.008172 \\
& SELF & 0.254494 & 0.002910 & 0.250817 & 0.008561 & 0.718261 & 0.005899 \\
& QLF & 0.232193 & 0.045345 & 0.187496 & 0.141003 & 0.700214 & 0.013121 \\
& WLF & 0.243222 & 0.011227 & 0.218273 & 0.032544 & 0.709524 & 0.008736 \\
& DLF & 0.265888 & 0.043022 & 0.284952 & 0.119793 & 0.726475 & 0.011306 \\
& PLF & 0.260106 & 0.011313 & 0.267341 & 0.033046 & 0.722356 & 0.008191 \\
\hline
\end{tabular}

Table 3 BEs and PRs using Jeffreys prior.

\begin{tabular}{llcccccc}
\hline \multirow{2}{*}{$t_{0}=73$} & & $\boldsymbol{\theta}_{\mathbf{1}}$ & $\boldsymbol{p}\left(\boldsymbol{\theta}_{\mathbf{1}}\right)$ & $\boldsymbol{\theta}_{\mathbf{2}}$ & $\boldsymbol{p}\left(\boldsymbol{\theta}_{\mathbf{2}}\right)$ & $\boldsymbol{p}$ & $\boldsymbol{p}(\boldsymbol{p})$ \\
& SELF & 0.196161 & 0.002326 & 0.230747 & 0.022812 & 0.787767 & 0.007602 \\
& QLF & 0.174183 & 0.058031 & 0.194476 & 0.327727 & 0.765022 & 0.015449 \\
& WLF & 0.184914 & 0.011247 & 0.151778 & 0.078968 & 0.777260 & 0.010741 \\
& DLF & 0.208023 & 0.057022 & 0.329611 & 0.249942 & 0.797417 & 0.012101 \\
& PLF & 0.202005 & 0.011688 & 0.275784 & 0.090074 & 0.792577 & 0.009620 \\
& SELF & 0.246665 & 0.002841 & 0.221363 & 0.007256 & 0.714257 & 0.005972 \\
& QLF & 0.224241 & 0.047218 & 0.160968 & 0.133998 & 0.695907 & 0.013414 \\
& WLF & 0.235354 & 0.011310 & 0.190269 & 0.031094 & 0.705369 & 0.008873 \\
& DLF & 0.258184 & 0.044617 & 0.254143 & 0.118983 & 0.722618 & 0.011571 \\
& PLF & 0.252359 & 0.011388 & 0.237188 & 0.031648 & 0.718425 & 0.008337 \\
\hline
\end{tabular}

Table 4 BEs and PRs using gamma prior.

\begin{tabular}{llcccccc}
\hline \multicolumn{1}{c}{$t_{0}=73$} & & $\boldsymbol{\theta}_{\mathbf{1}}$ & $\boldsymbol{p}\left(\boldsymbol{\theta}_{\mathbf{1}}\right)$ & $\boldsymbol{\theta}_{\mathbf{2}}$ & $\boldsymbol{p}\left(\boldsymbol{\theta}_{\mathbf{2}}\right)$ & $\boldsymbol{p}$ & $\boldsymbol{p}(\boldsymbol{p})$ \\
& SELF & 0.206643 & 0.002629 & 0.427152 & 0.041871 & 0.824335 & 0.005374 \\
& QLF & 0.187974 & 0.056942 & 0.241486 & 0.346217 & 0.808934 & 0.016411 \\
& WLF & 0.197170 & 0.011184 & 0.330165 & 0.075375 & 0.817080 & 0.011251 \\
& DLF & 0.216465 & 0.056042 & 0.525175 & 0.280056 & 0.830855 & 0.012841 \\
& PLF & 0.211497 & 0.011621 & 0.473635 & 0.084925 & 0.827589 & 0.010067 \\
& SELF & 0.268992 & 0.003023 & 0.292001 & 0.009777 & 0.720680 & 0.005872 \\
& QLF & 0.247120 & 0.046291 & 0.228837 & 0.145588 & 0.702745 & 0.014094 \\
& WLF & 0.257959 & 0.011173 & 0.259714 & 0.030281 & 0.712003 & 0.009208 \\
& DLF & 0.280233 & 0.043781 & 0.325485 & 0.123185 & 0.728829 & 0.012136 \\
& PLF & 0.274555 & 0.011247 & 0.308289 & 0.030811 & 0.724743 & 0.008635 \\
\hline
\end{tabular}


Table 5 BEs and PRs using inverse levy prior.

\begin{tabular}{llcccccc}
\hline \multicolumn{1}{c}{$t_{0}=73$} & & $\boldsymbol{\theta}_{\mathbf{1}}$ & $\boldsymbol{p}\left(\boldsymbol{\theta}_{\mathbf{1}}\right)$ & $\boldsymbol{\theta}_{\mathbf{2}}$ & $\boldsymbol{p}\left(\boldsymbol{\theta}_{\mathbf{2}}\right)$ & $\boldsymbol{p}$ & $\boldsymbol{p}(\boldsymbol{p})$ \\
& SELF & 0.201720 & 0.002342 & 0.217076 & 0.021999 & 0.771811 & 0.003858 \\
& QLF & 0.179240 & 0.046640 & 0.100155 & 0.268589 & 0.747168 & 0.009969 \\
& WLF & 0.190208 & 0.009472 & 0.150569 & 0.096987 & 0.760161 & 0.007255 \\
& DLF & 0.213870 & 0.045375 & 0.298896 & 0.186648 & 0.782294 & 0.007487 \\
& PLF & 0.207707 & 0.009708 & 0.254722 & 0.092964 & 0.777035 & 0.006507 \\
& SELF & 0.248441 & 0.002826 & 0.226761 & 0.007224 & 0.705025 & 0.004106 \\
& QLF & 0.226284 & 0.042019 & 0.167875 & 0.118888 & 0.686009 & 0.013003 \\
& WLF & 0.237267 & 0.011032 & 0.196480 & 0.032287 & 0.695816 & 0.008677 \\
& DLF & 0.259816 & 0.040112 & 0.258619 & 0.102874 & 0.713687 & 0.011180 \\
& PLF & 0.254065 & 0.011125 & 0.242167 & 0.032575 & 0.709343 & 0.008125 \\
\hline
\end{tabular}

The results show that by increasing the censoring rate from $t_{0}=73$ to $t_{0}=100$, the estimates are closely estimated. The result confirms the fact that as the censoring rate increases indefinitely, the estimates are more accurate as well as the risks become minimum. The minimum risks are obtained using SELF for all priors. Among noninformative priors, JP provides minimum risk and comparison of all the priors shows that the risks for the parameter $\theta_{1}, \theta_{2}$ and $p$ are minimum for ILP.

\subsection{Real-Life Application}

Gupta et al. [16] consider the two data sets of successive failure time (in hours) of the air conditioning system of two jet planes, plane8044 and plane-7912, consisting of 12 and 30 observations, respectively, and fit these data sets to LD. The strength of data shows mixing proportion of first component to be about 0.714 . For different censoring rates with fixed mixing proportion, the division of data set is as follows:

\begin{tabular}{lllll}
\hline$p=0.714$ & $n_{1}=30$ & $t_{0}=73$ & $r_{1}=22$ & $r_{2}=5$ \\
& $n_{2}=12$ & $t_{0}=100$ & $r_{1}=25$ & $r_{2}=9$ \\
\hline
\end{tabular}

The results are tabulated in an Appendix from Tables A1-A10 which clearly show BEs and PRs for different priors under SELF, WLF, QLF, PLF and DLF are consistent with the results given in Gupta et al. [16] using similar sampled data. Here for the sake of convenience we only present computed results based on Uniform and Jeffreys priors. Other results can be obtained from corresponding author on request.

\section{CONCLUDING REMARKS}

The simulation study reveals some interesting properties regarding BEs and PRs. The BEs of different parameters approaches to their true values as the sample size increases and the PRs of the estimates decreases with an increase in sample size. For smaller sample sizes, estimates of $\theta_{1}$ are over estimated and the estimates of $\theta_{2}$ and $p$ are underestimated but the sampling error approaches zero as the sample size increases. For larger parameter values (assumed) the risks are also relatively large as compared to the risks of small parametric values. Also, the PRs for $\theta_{1}$ and $p$ decreases with the increase in mixing proportion but the risk for $\theta_{2}$ increases with the increase in the value of mixing proportion.

For the different cases $\left(\theta_{1}<\theta_{2}\right),\left(\theta_{1}>\theta_{2}\right),\left(\theta_{1}=\theta_{2}\right)$ we discussed, it is obvious that in all the cases degree of over and under estimation reduces with the increase of mixing proportion. By increasing the censoring rate, the estimates are comparatively more accurate and precise with low PR in case of smaller sample size. For larger sample size the effect of censoring and mixing proportion almost vanishes. When censoring rate $\left(t_{0}\right)$ approaches infinity, the effect of censoring vanishes thus the sample no more remains incomplete. Consequently, the expressions for the BEs and the PRs get more simplified and the estimators are estimated with high precision with PRs expected to be smaller.

Over estimation and underestimation decreases with the increase of censoring rate for all the parameters. But increasing the mixing proportion parameter leads to decreasing the overestimation of first component parameter and increasing the underestimation of second component parameter. Whereas the estimates of proportion parameter itself are precisely estimated for large values of mixing proportion and censoring rate.

Considering the non-informative priors, estimates by Jeffrey's prior are more precise with high efficiency. And among the informative priors, the estimates are more precisely estimated by Inverse Levy prior also the PRs are minimum. For the case when both component parameters are equal QLF provides least variances. When first component parameter is less than the second then SELF gives least PR and when first component parameter is greater than the second then DLF provides minimum risk for both priors. 
Also, the results of real data set show that the estimates are more precisely estimated using Inverse Levy prior and the PRs are minimum using SELF for Inverse Levy Prior for all parameters. Thus, from all above evidences it is suggested that for the two-component mixture of LD, Inverse Levy prior is more preferable and choicer of loss function depends on values of $\theta_{1}$ and $\theta_{2}$. Due to these mix results we only present in Appendix (Tables A1-A10), only Uniform and Jeffreys priors results. The rest of the results for inverse levy priors and Gamma priors can be obtained from the authors on request.

\section{CONFLICTS OF INTEREST}

The authors declare they have no conflicts of interest.

\section{AUTHORS' CONTRIBUTIONS}

Authors' equally contributed to the paper under supervision of Prof Aslam.

\section{ACKNOWLEDGMENTS}

We acknowledge with thanks the constructive comments provided by two anonymous referees and the editor which improve the presentation of the paper. We're responsible of any errors.

\section{REFERENCES}

1. S. Ali, Appl. Math. Model. 39 (2015), 515-530.

2. M. Aslam, J. Stat. Theory Appl. 2 (2003), 70-83.

3. A.L. Corbellini, L. Crosato, P. Ganugi, M. Mazzoli, in: C. Skiadas (Ed.), Advances in Data Analysis, Birkhäuser, Boston, MA, USA, 2010.

4. M.Y. Majeed, M. Aslam, Int. J. Phys. Sci. 7 (2012), 1424-1434.

5. T. Sultana, M. Aslam, J. Shabbir, J. Adv. Math. Comput. Sci. 31 (2019), 1-16.

6. R.A. ZeinEldin, M. Ahsan ul Haq, S. Hashmi, M. Elsehety, Complexity. 2020 (2020), 1-15.

7. E.S.A. El-Sherpieny, E.M. Almetwally, H.Z. Muhammed, Physica A Stat. Mech. Appl. 553 (2020), 124251.

8. M. Ibrahim, H.M. Yousof, J. Data. Sci. 18 (2020), 190-217.

9. T. Sultana, M. Aslam, M. Raftab, J. Natl. Sci. Found. Sri Lanka. 45 (2017), 287-306.

10. K.S. Lomax, J. Am. Stat. Assoc. 49 (1954), 847-852.

11. A.S. Hassan, A.S. Al-Ghamdi, J. Appl. Sci. Res. 5 (2009), 2153-2164.

12. C.M. Harris, ROper. Res. 16 (1968), 307-313.

13. O.A. Holland, A. Golaup, A.H. Aghvami, IEEE Proc. Commun. 153 (2006), 683-690.

14. E. Cramer, A.B. Schmiedt, Comput. Stat. Data Anal. 55 (2011), 1285-1303.

15. D.E. Giles, H. Feng, R.T. Godwin, On the Bias of the Maximum Likelihood Estimator for the Two-Parameter Lomax Distribution, Econometrics Working Paper EWP1104, University of Victoria (Department of Economics), Victoria, BC, Canada, 2011. ISSN 1485-6441.

16. R.C. Gupta, M.E. Ghitany, M.E. Al-Mutairi, J. Stat. Comput. Simul. 80 (2010), 937-947.

17. B. Panathumparambath, Probstat Forum. 4 (2011), 1-11.

18. J.D. Kalbfleisch, R.L. Prentice, The Statistical Analysis of Failure Time Data, John Wiley \& Sons, New York, USA, 2011.

19. A. Shrivastava, A. Chaturvedi, M.I. Bhatti, Physica A Stat. Mech. Appl. 528 (2019), 121451.

20. M.I. Bhatti, H. Al-Shanfari, M.Z. Hossain, Econometric Analysis of Model Selection and Model Testing, Ashgate Publishing, Ltd., London, 2006.

21. W. Mendenhall, A.R. Hader, Biometrika. 45(3/4) (1958), 504-520.

22. A. Legendre, Nouvelles Methods Pour la Determination des Orbites des Cometes, Courcier, Paris, France, 1805.

23. C.F. Gauss, Method des Moindres Carres Memoire sur la Combinaison des Observations, 1810, Translated by J. Bertrand, Mallet-Bachelier, Paris, France, 1995.

24. J.G. Norstorm, IEEE Trans. Reliab. 45 (1996), 400-403. 


\section{APPENDIX}

This appendix contains some selected priors simulation results of BEs, PRs for SELF, QLF, WLF, DLF and PLF for various priors for 30, 100, 500, 1000 samples.

Table A1 BEs and PRs using UNIFORM PRIOR under $\mathrm{L}_{1}$ (SELF).

\begin{tabular}{|c|c|c|c|c|c|c|}
\hline & $\theta_{1}$ & $p\left(\theta_{1}\right)$ & $\theta_{2}$ & $p\left(\theta_{2}\right)$ & $p$ & $p(p)$ \\
\hline & & $t_{0}=2$ & $\theta_{1}=0.5$ & $\theta_{2}=1$ & & \\
\hline 30 & 0.868198 & $(0.165876)$ & 0.566810 & $(0.047911)$ & 0.327811 & $(0.017896)$ \\
\hline 100 & 0.549236 & $(0.055755)$ & 0.932172 & $(0.018459)$ & 0.367294 & $(0.011056)$ \\
\hline 500 & 0.533101 & $(0.019924)$ & 0.931439 & $(0.012612)$ & 0.392734 & $(0.002602)$ \\
\hline 1000 & 0.501517 & $(0.011194)$ & 1.001905 & $(0.009384)$ & 0.400928 & $(0.001882)$ \\
\hline & & $t_{0}=6$ & $\theta_{1}=0.5$ & $\theta_{3}=1$ & & \\
\hline 30 & 0.675280 & $(0.073515)$ & 0.791838 & $(0.071218)$ & 0.569459 & $(0.017918)$ \\
\hline 100 & 0.583011 & $(0.025001)$ & 0.910828 & $(0.042682)$ & 0.676756 & $(0.008942)$ \\
\hline 500 & 0.522339 & $(0.003327)$ & 0.955894 & $(0.021318)$ & 0.686672 & $(0.002527)$ \\
\hline 1000 & 0.520646 & $(0.000872)$ & 1.070055 & $(0.019874)$ & 0.701941 & $(0.001586)$ \\
\hline & & $t_{0}=2$ & $\theta_{1}=5$ & $\theta_{2}=3$ & & \\
\hline 30 & 7.604810 & $(0.991806)$ & 2.554840 & $(0.561905)$ & 0.406250 & $(0.007309)$ \\
\hline 100 & 6.753140 & $(0.342834)$ & 2.922890 & $(0.094706)$ & 0.401961 & $(0.002338)$ \\
\hline 500 & 5.622758 & $(0.120436)$ & 2.974216 & $(0.027980)$ & 0.400398 & $(0.000477)$ \\
\hline 1000 & 5.148023 & $(0.060119)$ & 3.006162 & $(0.014024)$ & 0.400234 & $(0.000239)$ \\
\hline & & $t_{0}=6$ & $\theta_{1}=5$ & $\theta_{2}=3$ & & \\
\hline 30 & 5.985530 & $(0.923211)$ & 2.661400 & $(0.717968)$ & 0.687500 & $(0.006510)$ \\
\hline 100 & 5.549090 & $(0.329613)$ & 2.896205 & $(0.113819)$ & 0.696078 & $(0.002053)$ \\
\hline 500 & 5.383147 & $(0.070854)$ & 2.995290 & $(0.060521)$ & 0.699203 & $(0.004181)$ \\
\hline 1000 & 5.160031 & $(0.030414)$ & 3.005316 & $(0.029221)$ & 0.699643 & $(0.000209)$ \\
\hline
\end{tabular}

Table A2 BEs and PRs using UNIFORM PRIOR under $\mathrm{L}_{2}(\mathrm{QLF})$.

\begin{tabular}{|c|c|c|c|c|c|c|c|}
\hline \multirow[b]{3}{*}{30} & \multirow[t]{2}{*}{$\theta_{1}$} & $p\left(\theta_{1}\right)$ & $\theta_{2}$ & \multicolumn{2}{|r|}{$p\left(\theta_{2}\right)$} & \multirow[t]{2}{*}{$p$} & \multirow[t]{2}{*}{$p(p)$} \\
\hline & & $t_{0}=2$ & $\theta_{\mathbf{1}}=\mathbf{0 . 5}$ & $\theta_{2}=1$ & $p=0.40$ & & \\
\hline & 0.632357 & $(0.175062)$ & 0.505066 & \multicolumn{2}{|r|}{$(0.149743)$} & 0.171839 & $(0.005801)$ \\
\hline 100 & 0.566879 & $(0.158769)$ & 0.802898 & & $(0.082497)$ & 0.334491 & $(0.087468)$ \\
\hline 500 & 0.509119 & $(0.104761)$ & 0.885876 & & $(0.019897)$ & 0.398347 & $(0.039793)$ \\
\hline \multirow[t]{2}{*}{1000} & 0.500947 & $(0.077323)$ & 1.005357 & & $(0.017473)$ & 0.396483 & $(0.006223)$ \\
\hline & & $t_{0}=6$ & $\theta_{1}=0.5$ & \multicolumn{3}{|c|}{$p=0.70$} & \\
\hline 30 & 0.591142 & $(0.058690)$ & 0.261905 & \multicolumn{2}{|r|}{$(0.419453)$} & 0.592556 & $(0.145618)$ \\
\hline 100 & 0.566278 & $(0.053876)$ & 0.671198 & & $(0.133678)$ & 0.652334 & $(0.058523)$ \\
\hline 500 & 0.553462 & $(0.010230)$ & 0.872834 & & $(0.101367)$ & 0.692696 & $(0.010113)$ \\
\hline \multirow[t]{2}{*}{1000} & 0.523607 & $(0.007475)$ & 0.980768 & & (0.047828) & 0.703447 & $(0.005824)$ \\
\hline & & $t_{0}=2$ & $\theta_{1}=5$ & $\theta_{2}=3$ & $p=0$ & & \\
\hline 30 & 6.496400 & $(0.083333)$ & 2.266020 & & $(0.055556)$ & 0.366667 & $(0.603113)$ \\
\hline 100 & 5.469900 & $(0.025000)$ & 2.771640 & & $(0.016667)$ & 0.390877 & $(0.015875)$ \\
\hline 500 & 5.314815 & $(0.005000)$ & 2.994140 & & $(0.003356)$ & 0.398000 & $(0.003034)$ \\
\hline 1000 & 5.043173 & $(0.002511)$ & 3.009386 & & $(0.001676)$ & 0.399041 & $(0.001508)$ \\
\hline & & $t_{0}=6$ & $\theta_{1}=5$ & $\theta_{2}=3$ & $p=$ & & \\
\hline 30 & 6.534900 & $(0.046719)$ & 2.503140 & & $(0.111111)$ & 0.666667 & $(0.018273)$ \\
\hline 100 & 5.287920 & $(0.014285)$ & 2.800430 & & $(0.035457)$ & 0.690000 & $(0.004633)$ \\
\hline 500 & 5.218393 & $(0.002883)$ & 2.814160 & & $(0.006667)$ & 0.698000 & $(0.000871)$ \\
\hline 1000 & 5.182866 & $(0.001447)$ & 2.982676 & & $(0.003405)$ & 0.699119 & $(0.000432)$ \\
\hline
\end{tabular}


Table A3 BEs and PRs using UNIFORM PRIOR under $\mathrm{L}_{3}$ (WLF).

\begin{tabular}{|c|c|c|c|c|c|c|}
\hline \multirow[b]{3}{*}{30} & \multirow[t]{2}{*}{$\theta_{1}$} & \multirow{2}{*}{$\begin{array}{c}p\left(\theta_{1}\right) \\
t_{0}=2\end{array}$} & $\theta_{2}$ & $p\left(\theta_{2}\right)$ & \multirow[t]{2}{*}{$p$} & \multirow[t]{2}{*}{$p(p)$} \\
\hline & & & $\theta_{1}=0.5$ & $\theta_{2}=1$ & & \\
\hline & 0.586696 & $(0.844801)$ & 0.861946 & $(0.505345)$ & 0.309341 & $(0.048654)$ \\
\hline 100 & 0.509670 & $(0.129690)$ & 0.951471 & $(0.134949)$ & 0.353419 & $(0.023179)$ \\
\hline 500 & 0.501270 & $(0.020554)$ & 0.965427 & $(0.027140)$ & 0.389600 & $(0.014713)$ \\
\hline \multirow[t]{2}{*}{1000} & 0.500387 & $(0.008816)$ & 1.030835 & $(0.009214)$ & 0.395952 & $(0.006961)$ \\
\hline & & $t_{0}=6$ & $\theta_{1}=\mathbf{0 . 5}$ & $\theta_{2}=1$ & $p=0.70$ & \\
\hline 30 & 0.781563 & $(0.276677)$ & 0.594523 & $(0.590961)$ & 0.609370 & $(0.022965)$ \\
\hline 100 & 0.571912 & $(0.134396)$ & 0.814298 & $(0.259899)$ & 0.689392 & $(0.013148)$ \\
\hline 500 & 0.544786 & $(0.042615)$ & 0.891128 & $(0.082066)$ & 0.694377 & $(0.006429)$ \\
\hline \multirow[t]{2}{*}{1000} & 0.516595 & $(0.003875)$ & 0.996003 & $(0.054302)$ & 0.699735 & $(0.002059)$ \\
\hline & & $t_{0}=2$ & $\theta_{1}=\mathbf{5}$ & $\theta_{2}=3$ & $p=0.40$ & \\
\hline 30 & 8.338230 & $(0.689299)$ & 2.314470 & $(0.137066)$ & 0.387097 & $(0.019153)$ \\
\hline 100 & 5.208370 & $(0.134820)$ & 2.801260 & $(0.047563)$ & 0.396040 & $(0.005921)$ \\
\hline 500 & 5.192357 & $(0.024575)$ & 2.898484 & $(0.009390)$ & 0.399258 & $(0.001196)$ \\
\hline \multirow[t]{2}{*}{1000} & 5.081537 & $(0.013255)$ & 2.976806 & $(0.004901)$ & 0.399634 & $(0.000599)$ \\
\hline & & $t_{0}=6$ & $\theta_{1}=\mathbf{5}$ & $\theta_{2}=3$ & $p=0.70$ & \\
\hline 30 & 6.253390 & $(0.221080)$ & 2.302440 & $(0.411727)$ & 0.677419 & $(0.010082)$ \\
\hline 100 & 5.076946 & $(0.081214)$ & 2.790350 & $(0.073253)$ & 0.693069 & $(0.003009)$ \\
\hline 500 & 5.067001 & $(0.013328)$ & 2.920909 & $(0.019690)$ & 0.698752 & $(0.000600)$ \\
\hline 1000 & 5.032640 & $(0.007037)$ & 2.992230 & $(0.009871)$ & 0.699351 & $(0.000301)$ \\
\hline
\end{tabular}

Table A4 BEs and PRs using UNIFORM PRIOR under $\mathrm{L}_{4}$ (DLF).

\begin{tabular}{|c|c|c|c|c|c|c|}
\hline \multirow[b]{3}{*}{30} & \multirow[t]{2}{*}{$\theta_{1}$} & $p\left(\theta_{1}\right)$ & $\theta_{2}$ & $p\left(\theta_{2}\right)$ & \multirow[t]{2}{*}{$p$} & \multirow[t]{2}{*}{$p(p)$} \\
\hline & & $t_{0}=2$ & $\theta_{1}=0.5$ & $\theta_{2}=1$ & & \\
\hline & 1.384370 & $(0.373828)$ & 0.804731 & $(0.136817)$ & 0.388311 & $(0.157103)$ \\
\hline 100 & 0.891284 & $(0.183368)$ & 0.932716 & $(0.073123)$ & 0.398584 & $(0.105112)$ \\
\hline 500 & 0.631031 & $(0.051093)$ & 0.965631 & $(0.020707)$ & 0.398906 & $(0.042611)$ \\
\hline \multirow[t]{2}{*}{1000} & 0.545555 & $(0.050509)$ & 1.029706 & $(0.023792)$ & 0.399319 & $(0.023323)$ \\
\hline & & $t_{0}=6$ & $\theta_{1}=0.5$ & $\theta_{2}=1$ & $p=0.70$ & \\
\hline 30 & 1.163020 & $(0.378258)$ & 0.758247 & $(0.668067)$ & 0.618951 & $(0.039674)$ \\
\hline 100 & 0.593345 & $(0.172703)$ & 0.909517 & $(0.244430)$ & 0.686122 & $(0.044180)$ \\
\hline 500 & 0.529034 & $(0.096319)$ & 0.973550 & $(0.117226)$ & 0.696445 & $(0.005753)$ \\
\hline \multirow[t]{2}{*}{1000} & 0.510351 & $(0.028826)$ & 0.992942 & $(0.055786)$ & 0.699411 & $(0.005037)$ \\
\hline & & $t_{0}=2$ & $\theta_{1}=5$ & $\theta_{2}=3$ & $p=0.40$ & \\
\hline 30 & 6.300940 & $(0.071428)$ & 2.543270 & $(0.050000)$ & 0.424242 & $(0.042410)$ \\
\hline 100 & 5.166071 & $(0.023809)$ & 2.820998 & $(0.016129)$ & 0.407767 & $(0.014239)$ \\
\hline 500 & 5.161960 & $(0.004950)$ & 2.967710 & $(0.003558)$ & 0.401618 & $(0.002968)$ \\
\hline \multirow[t]{2}{*}{1000} & 5.050240 & $(0.002496)$ & 3.000653 & $(0.001667)$ & 0.400747 & $(0.001492)$ \\
\hline & & $t_{0}=6$ & $\theta_{1}=\mathbf{5}$ & $\theta_{2}=3$ & $p=0.70$ & \\
\hline 30 & 6.838610 & $(0.043478)$ & 1.894940 & $(0.090909)$ & 0.696970 & $(0.013587)$ \\
\hline 100 & 5.208830 & $(0.013888)$ & 2.972160 & $(0.031250)$ & 0.699029 & $(0.004221)$ \\
\hline 500 & 5.168281 & $(0.002841)$ & 2.995387 & $(0.006578)$ & 0.699801 & $(0.000854)$ \\
\hline 1000 & 5.096793 & $(0.001431)$ & 3.050068 & $(0.003343)$ & 0.699979 & $(0.000427)$ \\
\hline
\end{tabular}


Table A5 BEs and PRs using UNIFORM PRIOR under $\mathrm{L}_{5}$ (PLF).

\begin{tabular}{|c|c|c|c|c|c|c|}
\hline & $\theta_{1}$ & $p\left(\theta_{1}\right)$ & $\theta_{2}$ & $p\left(\theta_{2}\right)$ & $p$ & $p(p)$ \\
\hline & & $t_{0}=2$ & $\theta_{1}=0.5$ & $\theta_{2}=1$ & & \\
\hline 30 & 3.029090 & $(0.576995)$ & 0.904122 & $(0.693269)$ & 0.340176 & $(0.059611)$ \\
\hline 100 & 0.748465 & $(0.240711)$ & 0.929811 & $(0.101780)$ & 0.375614 & $(0.080096)$ \\
\hline 500 & 0.665705 & $(0.086592)$ & 0.978891 & $(0.017721)$ & 0.389919 & $(0.009907)$ \\
\hline 1000 & 0.532694 & $(0.022393)$ & 0.999222 & $(0.023022)$ & 0.399967 & $(0.007601)$ \\
\hline & & $t_{0}=6$ & $\theta_{1}=0.5$ & $\theta_{2}=1$ & & \\
\hline 30 & 1.975590 & $(0.370766)$ & 0.764774 & $(0.908008)$ & 0.623231 & $(0.068635)$ \\
\hline 100 & 0.667103 & $(0.031672)$ & 0.889489 & $(0.231578)$ & 0.674139 & $(0.020084)$ \\
\hline 500 & 0.589359 & $(0.027025)$ & 0.946213 & $(0.092694)$ & 0.685368 & $(0.002013)$ \\
\hline 1000 & 0.532825 & $(0.002759)$ & 0.990916 & $(0.055924)$ & 0.707922 & $(0.007072)$ \\
\hline & & $t_{0}=2$ & $\theta_{1}=5$ & $\theta_{2}=3$ & & \\
\hline 30 & 5.956680 & $(0.428334)$ & 2.449380 & $(0.117465)$ & 0.415149 & $(0.017797)$ \\
\hline 100 & 5.159670 & $(0.109574)$ & 2.748210 & $(0.052718)$ & 0.405174 & $(0.055785)$ \\
\hline 500 & 5.141909 & $(0.024606)$ & 2.936234 & $(0.009137)$ & 0.401073 & $(0.001192)$ \\
\hline 1000 & 5.034269 & $(0.012646)$ & 3.067972 & $(0.004727)$ & 0.400523 & $(0.000597)$ \\
\hline & & $t_{0}=6$ & $\theta_{1}=5$ & $\theta_{2}=3$ & & \\
\hline 30 & 6.078330 & $(0.318649)$ & 2.539390 & $(0.315203)$ & 0.692219 & $(0.009437)$ \\
\hline 100 & 5.233460 & $(0.060905)$ & 2.954720 & $(0.129226)$ & 0.697552 & $(0.002947)$ \\
\hline 500 & 5.126028 & $(0.014828)$ & 2.986707 & $(0.018945)$ & 0.699502 & $(0.000597)$ \\
\hline 1000 & 5.082839 & $(0.007300)$ & 2.990327 & $(0.009915)$ & 0.699750 & $(0.000299)$ \\
\hline
\end{tabular}

Table A6 BEs and PRs using JEFFREYS PRIOR under $\mathrm{L}_{1}$ (SELF).

\begin{tabular}{|c|c|c|c|c|c|c|}
\hline \multirow[b]{3}{*}{30} & \multirow[t]{2}{*}{$\theta_{1}$} & \multirow{2}{*}{$\begin{array}{c}p\left(\theta_{1}\right) \\
t_{0}=2\end{array}$} & $\theta_{2}$ & $p\left(\theta_{2}\right)$ & \multirow[t]{2}{*}{$p$} & \multirow[t]{2}{*}{$p(p)$} \\
\hline & & & $\theta_{1}=\mathbf{0 . 5}$ & $\theta_{2}=1$ & & \\
\hline & 0.585082 & $(0.135402)$ & 0.723052 & $(0.519567)$ & 0.273948 & $(0.028809)$ \\
\hline 100 & 0.564843 & $(0.048855)$ & 0.858892 & $(0.190453)$ & 0.355601 & $(0.010338)$ \\
\hline 500 & 0.522329 & $(0.046113)$ & 0.875868 & $(0.041168)$ & 0.378890 & $(0.002988)$ \\
\hline \multirow[t]{2}{*}{1000} & 0.513741 & $(0.031783)$ & 0.995097 & $(0.012879)$ & 0.396673 & $(0.002948)$ \\
\hline & & $t_{0}=6$ & $\theta_{\mathbf{1}}=\mathbf{0 . 5}$ & \multicolumn{2}{|c|}{$p=0.70$} & \\
\hline 30 & 0.880612 & $(0.296853)$ & 0.722330 & $(0.179131)$ & 0.552438 & $(0.030204)$ \\
\hline 100 & 0.605321 & $(0.031509)$ & 0.910005 & $(0.076469)$ & 0.614797 & $(0.012708)$ \\
\hline 500 & 0.564290 & $(0.012527)$ & 0.959278 & $(0.052983)$ & 0.647509 & $(0.002701)$ \\
\hline \multirow[t]{2}{*}{1000} & 0.528352 & $(0.001525)$ & 0.979681 & $(0.038486)$ & 0.710503 & $(0.003162)$ \\
\hline & & $t_{0}=2$ & $\theta_{1}=5$ & $\theta_{2}=3$ & & \\
\hline 30 & 6.559450 & $(0.952253)$ & 2.365220 & $(0.612373)$ & 0.406250 & $(0.007309)$ \\
\hline 100 & 5.800614 & $(0.683055)$ & 2.886410 & $(0.191055)$ & 0.402058 & $(0.002333)$ \\
\hline 500 & 5.164538 & $(0.164619)$ & 2.900881 & $(0.033207)$ & 0.400506 & $(0.000477)$ \\
\hline 1000 & 5.053912 & $(0.064510)$ & 2.975125 & $(0.017154)$ & 0.400522 & $(0.000239)$ \\
\hline & & $t_{0}=6$ & $\theta_{1}=5$ & $\theta_{2}=3$ & & \\
\hline 30 & 5.625410 & $(0.855126)$ & 2.688910 & $(0.855126)$ & 0.687500 & $(0.000651)$ \\
\hline 100 & 5.244240 & $(0.448580)$ & 2.889040 & $(0.261109)$ & 0.696078 & $(0.002053)$ \\
\hline 500 & 5.227993 & $(0.066405)$ & 2.945624 & $(0.056815)$ & 0.699359 & $(0.000418)$ \\
\hline 1000 & 5.152844 & $(0.035136)$ & 3.013584 & $(0.028999)$ & 0.699640 & $(0.000209)$ \\
\hline
\end{tabular}


Table A7 BEs and PRs using JEFFREYS PRIOR under $\mathrm{L}_{2}$ (QLF).

\begin{tabular}{|c|c|c|c|c|c|c|}
\hline \multirow[b]{3}{*}{30} & \multirow[t]{2}{*}{$\theta_{1}$} & $p\left(\theta_{1}\right)$ & $\theta_{2}$ & $p\left(\theta_{2}\right)$ & \multirow[t]{2}{*}{$p$} & \multirow[t]{2}{*}{$p(p)$} \\
\hline & & $t_{0}=2$ & $\theta_{1}=0.5$ & $\theta_{2}=1$ & & \\
\hline & 0.668822 & $(0.313101)$ & 0.791445 & $(0.093269)$ & 0.338187 & $(0.293941)$ \\
\hline 100 & 0.516394 & $(0.109889)$ & 0.878872 & $(0.046942)$ & 0.355669 & $(0.073711)$ \\
\hline 500 & 0.504195 & $(0.066512)$ & 0.956071 & $(0.013453)$ & 0.396026 & $(0.029581)$ \\
\hline \multirow[t]{2}{*}{1000} & 0.503277 & $(0.040247)$ & 1.003594 & $(0.020357)$ & 0.399537 & $(0.019148)$ \\
\hline & & $t_{0}=6$ & $\theta_{1}=0.5$ & \multicolumn{2}{|c|}{$p=0.70$} & \\
\hline 30 & 0.726527 & $(0.224800)$ & 0.615168 & $(0.330203)$ & 0.556238 & $(0.114821)$ \\
\hline 100 & 0.582858 & $(0.102558)$ & 0.871519 & $(0.188551)$ & 0.679914 & $(0.020672)$ \\
\hline 500 & 0.559331 & $(0.014379)$ & 0.902288 & $(0.131363)$ & 0.685231 & $(0.017313)$ \\
\hline \multirow[t]{2}{*}{1000} & 0.507780 & $(0.013377)$ & 0.922781 & $(0.052634)$ & 0.694740 & $(0.007253)$ \\
\hline & & $t_{0}=2$ & $\theta_{1}=5$ & $\theta_{2}=3$ & & \\
\hline 30 & 5.362800 & $(0.090909)$ & 2.551690 & $(0.058823)$ & 0.366667 & $(0.052777)$ \\
\hline 100 & 5.136830 & $(0.026398)$ & 2.917830 & $(0.016942)$ & 0.390000 & $(0.015250)$ \\
\hline 500 & 5.127830 & $(0.005079)$ & 2.981360 & $(0.063378)$ & 0.398000 & $(0.003009)$ \\
\hline 1000 & 5.118275 & $(0.002514)$ & 3.015314 & $(0.001678)$ & 0.399000 & $(0.001502)$ \\
\hline & & $t_{0}=6$ & $\theta_{1}=5$ & $\theta_{2}=3$ & & \\
\hline 30 & 5.871640 & $(0.050000)$ & 2.317520 & $(0.125000)$ & 0.666667 & $(0.015873)$ \\
\hline 100 & 5.293600 & $(0.015674)$ & 2.901660 & $(0.038156)$ & 0.690000 & $(0.004428)$ \\
\hline 500 & 5.130910 & $(0.002913)$ & 2.986520 & $(0.006711)$ & 0.698082 & $(0.000862)$ \\
\hline 1000 & 5.015030 & $(0.001447)$ & 3.060204 & $(0.003385)$ & 0.699000 & $(0.000430)$ \\
\hline
\end{tabular}

Table A8 BEs and PRs using JEFFREYS PRIOR under $\mathrm{L}_{3}$ (WLF).

\begin{tabular}{|c|c|c|c|c|c|c|}
\hline \multirow[b]{3}{*}{30} & \multirow[t]{2}{*}{$\theta_{1}$} & \multirow{2}{*}{$\begin{array}{c}p\left(\theta_{1}\right) \\
t_{0}=2\end{array}$} & $\theta_{2}$ & $p\left(\theta_{2}\right)$ & \multirow[t]{2}{*}{$p$} & \multirow[t]{2}{*}{$p(p)$} \\
\hline & & & $\theta_{\mathbf{1}}=\mathbf{0 . 5}$ & $\theta_{2}=1$ & & \\
\hline & 0.590707 & $(0.194143)$ & 0.692371 & $(0.425679)$ & 0.292827 & $(0.069055)$ \\
\hline 100 & 0.557263 & $(0.109993)$ & 0.913592 & $(0.111282)$ & 0.351266 & $(0.055825)$ \\
\hline 500 & 0.516451 & $(0.024938)$ & 0.963975 & $(0.044147)$ & 0.394425 & $(0.011623)$ \\
\hline \multirow[t]{2}{*}{1000} & 0.504707 & $(0.018522)$ & 0.984169 & $(0.016986)$ & 0.398664 & $(0.007743)$ \\
\hline & & $t_{0}=6$ & $\theta_{1}=0.5$ & \multicolumn{2}{|c|}{$p=0.70$} & \\
\hline 30 & 0.626834 & $(0.138348)$ & 0.632403 & $(0.849189)$ & 0.578556 & $(0.433855)$ \\
\hline 100 & 0.547884 & $(0.085996)$ & 0.732918 & $(0.206329)$ & 0.656508 & $(0.041699)$ \\
\hline 500 & 0.521872 & $(0.025817)$ & 0.843924 & $(0.076572)$ & 0.699617 & $(0.004791)$ \\
\hline \multirow[t]{2}{*}{1000} & 0.501373 & $(0.005726)$ & 1.092504 & $(0.044093)$ & 0.699739 & $(0.001906)$ \\
\hline & & $t_{0}=2$ & $\theta_{1}=5$ & $\theta_{2}=3$ & & \\
\hline 30 & 6.324810 & $(0.638801)$ & 2.390590 & $(0.160987)$ & 0.387097 & $(0.019153)$ \\
\hline 100 & 5.340740 & $(0.137989)$ & 2.869770 & $(0.045798)$ & 0.396342 & $(0.005921)$ \\
\hline 500 & 5.266520 & $(0.026531)$ & 2.953811 & $(0.010765)$ & 0.399218 & $(0.001197)$ \\
\hline 1000 & 5.047764 & $(0.013367)$ & 3.012084 & $(0.005022)$ & 0.399678 & $(0.000599)$ \\
\hline & & $t_{0}=6$ & $\theta_{1}=5$ & $\theta_{2}=3$ & & \\
\hline 30 & 6.744220 & $(0.237071)$ & 2.383510 & $(0.472010)$ & 0.677419 & $(0.010081)$ \\
\hline 100 & 5.368690 & $(0.081721)$ & 2.927110 & $(0.096667)$ & 0.693069 & $(0.003009)$ \\
\hline 500 & 5.334866 & $(0.013489)$ & 2.945551 & $(0.019673)$ & 0.698602 & $(0.000601)$ \\
\hline 1000 & 5.055121 & $(0.007143)$ & 2.984116 & $(0.009555)$ & 0.699332 & $(0.003001)$ \\
\hline
\end{tabular}


Table A9 BEs and PRs using JEFFREYS PRIOR under $\mathrm{L}_{4}$ (DLF).

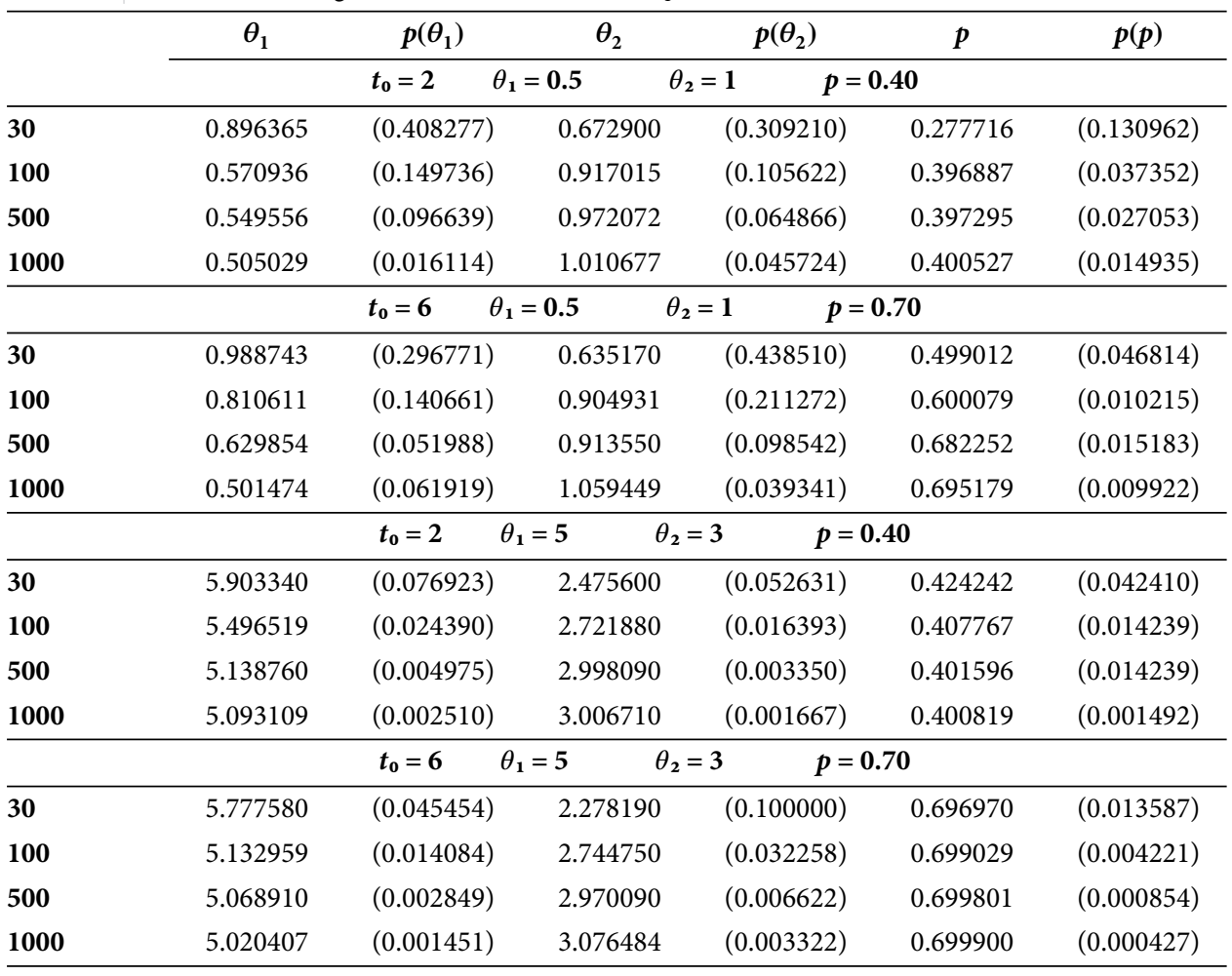

Table A10 BEs and PRs using JEFFREYS PRIOR under $\mathrm{L}_{5}$ (PLF).

\begin{tabular}{|c|c|c|c|c|c|c|}
\hline \multirow[b]{3}{*}{30} & \multirow[t]{2}{*}{$\theta_{1}$} & $p\left(\theta_{1}\right)$ & $\theta_{2}$ & $p\left(\theta_{2}\right)$ & \multirow[t]{2}{*}{$p$} & \multirow[t]{2}{*}{$p(p)$} \\
\hline & & $t_{0}=2$ & $\theta_{1}=0.5$ & $\theta_{2}=1$ & & \\
\hline & 0.939838 & $(0.924190)$ & 0.801237 & $(0.270134)$ & 0.305958 & $(0.074766)$ \\
\hline 100 & 0.645556 & $(0.111291)$ & 0.906830 & $(0.123313)$ & 0.352460 & $(0.033956)$ \\
\hline 500 & 0.522855 & $(0.032796)$ & 0.940166 & $(0.036917)$ & 0.373753 & $(0.006915)$ \\
\hline \multirow[t]{2}{*}{1000} & 0.515894 & $(0.033983)$ & 1.019609 & $(0.020426)$ & 0.401329 & $(0.004138)$ \\
\hline & & $t_{0}=6$ & $\theta_{1}=0.5$ & \multicolumn{2}{|c|}{$p=0.70$} & \\
\hline 30 & 0.720171 & $(0.913640)$ & 0.526735 & $(0.630469)$ & 0.600232 & $(0.032134)$ \\
\hline 100 & 0.593233 & $(0.059948)$ & 0.674720 & $(0.333007)$ & 0.678067 & $(0.027873)$ \\
\hline 500 & 0.550583 & $(0.052063)$ & 0.946675 & $(0.090064)$ & 0.686015 & $(0.019283)$ \\
\hline \multirow[t]{2}{*}{1000} & 0.509932 & $(0.003918)$ & 0.996137 & $(0.063856)$ & 0.697386 & $(0.004007)$ \\
\hline & & $t_{0}=2$ & $\theta_{1}=5$ & $\theta_{2}=3$ & & \\
\hline 30 & 6.903210 & $(0.248125)$ & 2.428070 & $(0.195582)$ & 0.415149 & $(0.017797)$ \\
\hline 100 & 5.406120 & $(0.166965)$ & 2.751020 & $(0.053699)$ & 0.404853 & $(0.005785)$ \\
\hline 500 & 5.341998 & $(0.025328)$ & 2.902147 & $(0.011029)$ & 0.400993 & $(0.001191)$ \\
\hline 1000 & 5.005876 & $(0.011616)$ & 3.003990 & $(0.004986)$ & 0.400516 & $(0.000597)$ \\
\hline & & $t_{0}=6$ & $\theta_{1}=5$ & $\theta_{2}=3$ & & \\
\hline 30 & 5.798350 & $(0.325978)$ & 2.636470 & $(0.301543)$ & 0.692219 & $(0.009437)$ \\
\hline 100 & 5.392956 & $(0.082651)$ & 2.878610 & $(0.096012)$ & 0.697552 & $(0.002947)$ \\
\hline 500 & 5.205450 & $(0.015214)$ & 2.946596 & $(0.021235)$ & 0.699502 & $(0.000597)$ \\
\hline 1000 & 5.000095 & $(0.007803)$ & 2.976622 & $(0.009954)$ & 0.699750 & $(0.000299)$ \\
\hline
\end{tabular}

